\title{
Data bus modelling in city buses
}

\author{
Jarosław Jajczyk ${ }^{1, *}$, Wojciech Lorkiewicz \\ ${ }^{1}$ Wydział Elektryczny, Politechnika Poznańska, Piotrowo 3A, 60-965 Poznań, Poland
}

\begin{abstract}
The paper characterizes ICT networks used in urban buses. It describes the multiplex system manufactured by Continental VOD and the ELSY multicomputer system manufactured by Thoreb, Differences in the structure and functioning of both systems were presented. Based on the Thoreb system, a test station for the modelling of a complex data bus used in city buses has been designed and built.
\end{abstract}

\section{Introduction}

Information technology successfully enters different areas of our life. This is also true of areas connected with transportation. The progressive increase in traffic in urban agglomerations, observed in the last few years, encourages us to use public transport. The greatest benefits are seen in the introduction of public transport $[1,2,3]$. To make the passengers willing to use the urban transport system, it must provide them with several amenities. At present, the manufactured buses are equipped with a heating and air conditioning systems, audio-visual systems which provide information to the passengers, monitoring systems, ticket punchers, door controllers, passenger counting systems, USB ports which allow for the charging of mobile devices, lighting systems and Internet Access Points.. The interconnection of such a large number of devices is provided using computer networks and multiplex systems. They are based on the use of a computer, the control and diagnostic capacities of which are extended by devices called multiplexers. A multiplexer allows for an increase in the number of computer inputs and outputs. The communication between a computer and a multiplexer is most often executed with the use of a wire transmission medium. The connection of elements in the network is based on one of the known vehicle communication protocols - most frequently a CAN bus $[4,5,6]$.

The design and manufacture of buses with the complex ICT structure is a difficult task which requires a whole range of tests and simulations. In such situations, systems for the modelling, simulation and testing of individual components and the entire system of the vehicle prove their usefulness. This paper presents a model of a multiplex network in a city bus.

\section{Multiplex systems in vehicles}

City buses among others are provided with systems connected with the passenger space (monitoring, ticket puncher system, passenger information system, door controllers or passenger counting system (Fig. 1)). Those systems are integrated and controlled by one, coherent multiplex system. The central unit communicates with the modules (multiplexes), which directly control the connected devices, via a CAN bus. Among the largest multiplex system manufacturers, one may list Continental VDO, Actia or Thoreb [7, 8, 9].

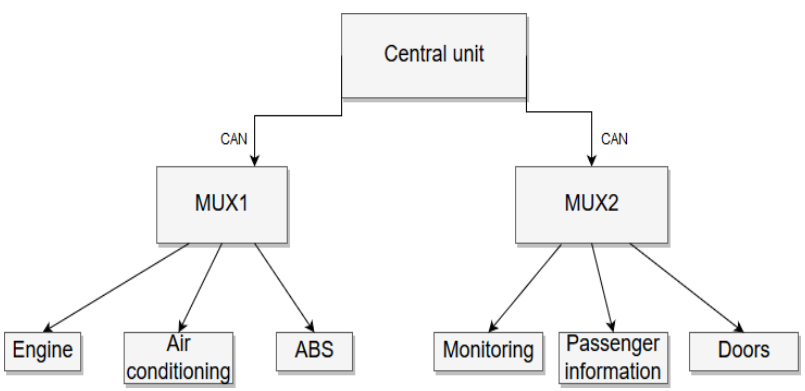

Fig. 1. Example of a structure of the bus multiplex system $[7,8]$

Several subsystems can be distinguished in a CAN bus of an urban bus, including systems responsible for the vehicle functions, e.g. PCAN (Powertrain CAN), ICAN (Instrumental CAN) or BCAN (Body CAN). The bus is controlled by a central processing unit, at the heart of which there is a microprocessor which executes instructions according to an uploaded software. A multiplexer is an element of the network, controlled by the central processing unit via the CAN bus. Owing to this, there is no need to have complex bundles of cables running to the central processing unit, which reduces the complexity of installation and its cost [5-9].

\subsection{CONTINENTAL VDO Kibes32 system}

The Kibes32 system from Continental VDO is a solution based on multiplexers. It consists of the ZR32-A central processing unit and MUX multiplexers (Fig. 2).

The computer has $6 \mathrm{CAN}$ interfaces and enables the connection of up to 16 multiplexers. Its calculating power is defined by two 32-bit processors, which work independently. It complies with the SAE J1708/J1587 protocol (a communication standard used e.g. in trucks). 


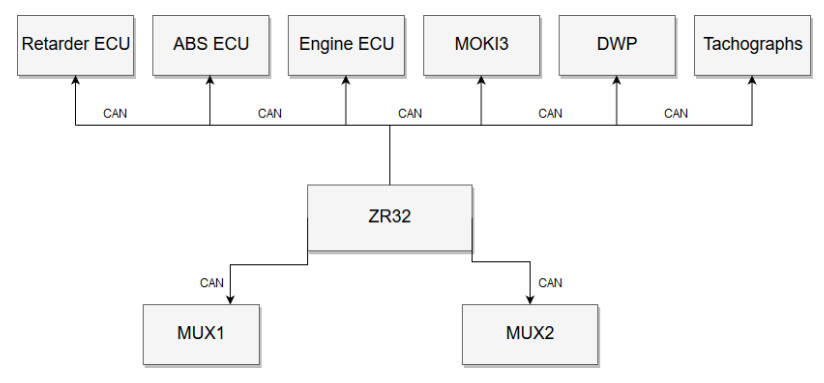

Fig. 2. Schematic diagram of Continental VDO system [7].

The diagnosis and programming are carried out via K-Line $[7,8]$.

\subsection{Thoreb ELSY system}

The ELSY system from the Swedish company Thoreb, the first version of which was developed already in 1986, is an alternative for the VDO multiplex system [8]. Contrary to the multiplex systems, Thoreb applies a different approach to the multi system control process. Instead of multiplexers and one computer, the system comprises of several equivalent computers connected via one common CAN bus. The use of several computers increases the reliability of the system in the case of a computer's failure and allows for the further operation of the whole system. The exemplary layout of computers in a bus is presented in Figure 3 [9, 10].

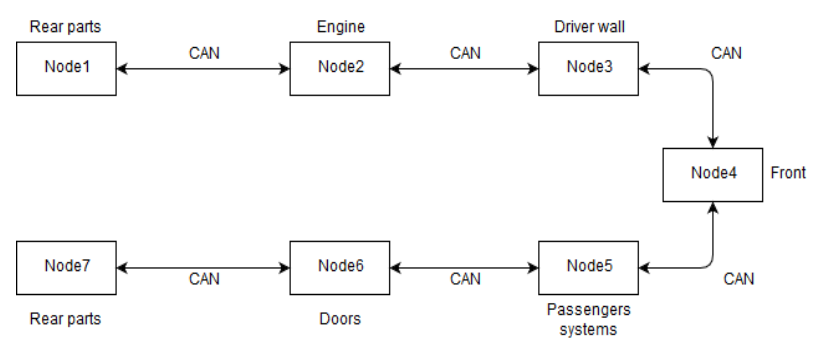

Fig. 3. Exemplary configuration of the ELSY system [9].

\section{The model of a bus multiplex system}

As part of the study, a test station based on the Thoreb system has been designed and built [9]. The developed solution ensures functionality which is similar to systems installed in buses. The station comprises of (Figure 4):

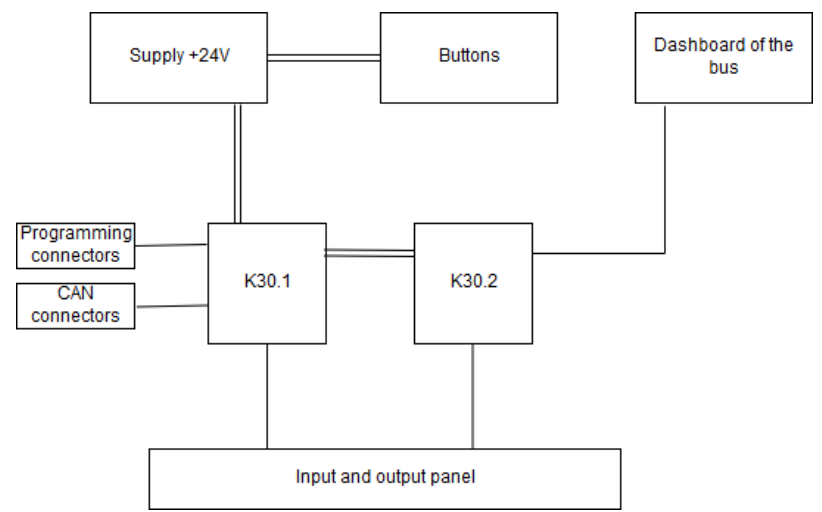

Fig. 4. Block diagram of the test station.
Thoreb K30 computers (connected via a CAN bus), $+24 \mathrm{~V}$ power supply, function buttons (KL15, desktop control), bus dashboard panel, connections used to program the computers and desktop, CAN bus connectors, the panel of input and output signal connectors (with LED diodes) [11].

As part of the study two programs controlling the selected devices (a three-way valve and a LED lamp) have been developed. The tests have proved correct functioning of the station. The communication between the computers is faultless and the inputs and outputs are correctly commanded [11].

\section{Conclusions}

Multiplex systems are complex systems which allow for the integration of independent controllers using a network which conforms with the CAN standard. The advantage of this architecture is that it is possible to diagnose the systems without interfering into the electrical system, and to freely program the system. The multiplex systems are popular among the manufacturers of city buses.

The performed studies and tests have allowed for the verification the behaviour of a multiplex system in different conditions. The station helped to analyse the behaviour of the communication bus of two computers by testing different variants of programs and performing a simultaneous analysis of the physical operation of the device (e.g. the signal condition switching rate). The built station allows for the test of the compatibility of the new solution with the existing bus system and the new software in a research laboratory. Advanced simulations performed on the test station save time and costs connected with the development of a reliable bus control software.

\section{References}

1. A. Dobrzycki. M. Filipiak, J. Jajczyk, Pozn Univ Technol Acad J, Electr Eng 92, 25-35 (2017)

2. J. Jajczyk, A. Dobrzycki, M. Filipiak, D. Kurz, E3S Web Conf. 1901027 (2017)

3. L. Kasprzyk, Eksploat Niezawodn 19 (2), 229-236 (2017)

4. A. Herner, H. Riehl, Elektrotechnika i elektronika w pojazdach samochodowych, WKiE, (2013)

5. M. Frei, Samochodowe magistrale danych $w$ praktyce warsztatowej, WKiŁ (2016)

6. J. Jajczyk, K. Matwiejczyk, Pozn Univ Technol Acad J, Electr Eng 79, 31-39 (2014)

7. Continental VDO catalog notice.

8. Kibes-32 Software Documentation ZR32-A.

9. Technical documentation of Thoreb computers.

10. Educational materials of the ElsyGraf program

11. J. Jajczyk, W. Lorkiewicz, Pozn Univ Technol Acad J, Electr Eng. 95, 321-332 (2018) 